\title{
Mobility-as-a-Service research trends of 5G-based vehicle platooning
}

\author{
Lingling $\mathrm{Lv}^{1} \cdot$ Yanjun Shi ${ }^{1} \cdot$ Weiming Shen ${ }^{2}$ \\ Published online: 26 November 2020 \\ (c) Springer-Verlag London Ltd., part of Springer Nature 2020
}

\section{Introduction}

Mobility-as-a-Service (MaaS) is often described as a travel payment platform that relies on existing means of transportation, including subways, buses, private cars, shared cars, shared bikes, and tailored mobility solutions catered to users' travel needs [1,2]. The uptake of MaaS will save time and cost, reduce congestion and traffic accidents. Previous studies proposed some services of MaaS: (1) customized solutions for individual travelers; (2) publicly commandeered private cars and shared cars for ridesharing; (3) automated vehicle (truck) platoons for transporting goods. According to an Allied Market Research report [3], the global truck platooning market size is projected to reach 4.59 billion US dollars by 2025 . This short article discusses some MaaS research trends of vehicle platooning with $5 \mathrm{G}$ technologies.

High-speed compact vehicle platoons can lead to higher road capacity, reduced fuel consumption and improved air quality than driving individually [4]. Wireless communication technologies (such as DSRC) can help platoons improve traffic safety and increase traffic flow throughput. With onboard units (OBU), connected and automated vehicles (CAVs) can reduce the driver's perception-reaction time and improve safety. With the help of vehicular communication, $\mathrm{CAV}$ s can collect information from other vehicles and roadside units (RSUs) and coordinate with other CAVs to control and manage the platoon, such as merge, split, and maintain a particular gap. The on-going development of OBU, RSU, etc., enables and enhances the MaaS of vehicle platooning.

Existing platoon-oriented CAVs have some limitations on onboard computation, sensor integration, and Vehicle-toeverything (V2X) communication, which cannot be managed by today's mobile communication infrastructures. $5 \mathrm{G}$ net-

\footnotetext{
Weiming Shen

wshen@ieee.org

1 School of Mechanical Engineering, Dalian University of Technology, Dalian 116024, China

2 State Key Lab of Digital Manufacturing Equipment and Technology, Huazhong University of Science and Technology, Wuhan 430074, China
}

works are expected to overcome these limitations. For example, 5G networks support multiple network slices, including enhanced mobile broadband (eMBB), massive machine-type communications (mMTC) and ultra-reliable low-latency communications (URLLC) to ensure the quality of service (QoS). URLLC guarantees safety for automated vehicles [5]. eMBB and URLLC can provide delay-sensitive services when massive vehicles are connected. Speed adjustments in a platoon can be made cooperatively through a $5 \mathrm{G}$ network. Vehicles share their kinematic information in real-time and negotiate speed adjustments to ensure efficient platooning formation and safety.

\section{MaaS trends of platooning}

This article proposes a MaaS architecture in 5G V2X networks (shown in Fig. 1) with some key technologies on MaaS for platooning orchestration. 5G, CAVs, electric vehicles, digital transportation infrastructure, cloud computing, mobile edge computing and big data analytics are all closely intertwined with MaaS in the future. Their trends are described in Mobility-as-a-Service, 5G V2X technologies, vehicle platooning, cloud-edge collaboration, and service scheduling.

(1) Mobility-as-a-Service

Technological enablers like 5G, Big Data, Artificial Intelligence and New IT infrastructure are expected to pave the way for a revolution in cities and inter-cities' mobility, enabling new MaaS for vehicle platooning. To design and develop a set of technology enablers that will empower the future MaaS, we envision the following directions as research trends: (1) 5G connectivity and cybersecurity; (2) MaaS services for platooning orchestration; and (3) collaborative computation on the cloud, edges and devices. These studies will enable the deployment of new services, requiring low latency and in-network processing. They will increase vehicle platooning efficiency by providing capabilities such as 


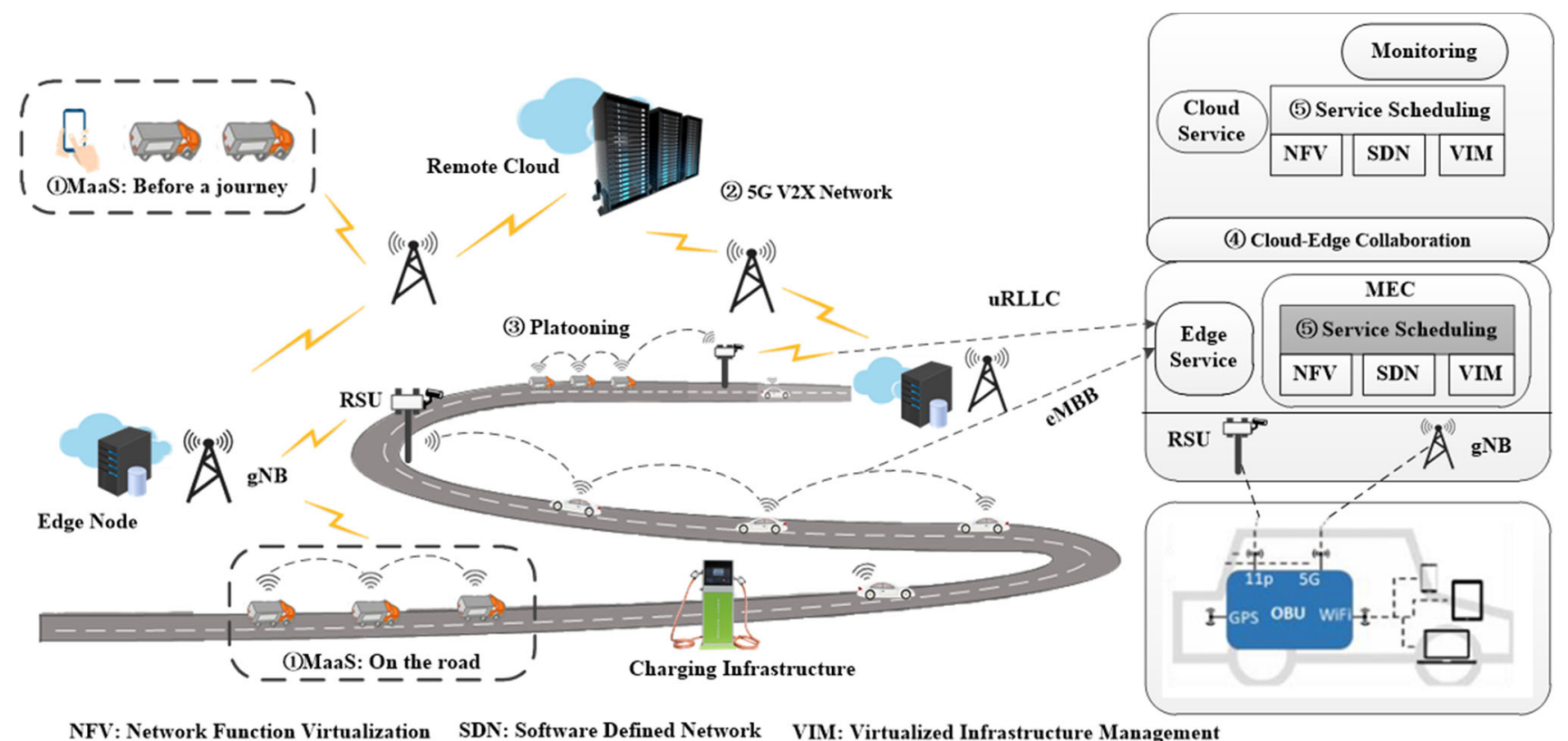

Fig. 1 A MaaS architecture in 5G V2X network

route planning before a trip or vehicle-to-vehicle coordination on the road.

(2) $5 \mathrm{G} \mathrm{V} 2 \mathrm{X}$ technologies

$\mathrm{V} 2 \mathrm{X}$ refers to vehicle-to-everything wireless communications, including Vehicle to Vehicle $(\mathrm{V} 2 \mathrm{~V})$, Vehicle to Infrastructure (V2I), Vehicle to Pedestrian (V2P), and Vehicle-to-Network (V2N), namely passing information from a vehicle to any entity that may affect the vehicle, and vice versa. The information exchange among vehicles and other entities will provide vehicles with a complete sense of their surrounding environment to improve traffic safety. The Third Generation Partnership Project (3GPP) discussed LTE-V evolutions in Release 15 to support 5G V2X (also known as LTE$\mathrm{V}$, LTE-V2X, or cellular V2X) communications and autonomous vehicles applications [6]. Two radio interfaces are included in the 5G V2X standard. The cellular interface (named $\mathrm{Uu}$ ) supports V2I communications, while the PC5 interface supports V2V communications based on direct LTE sidelink. 5G V2X supports highly reliable and low-latent V2X communications (URLLC slice), and thus deploying and using the 5G slices (URLLC or others) will be a trend for MaaS of vehicle platooning.

(3) Vehicle platooning

Vehicle platooning has become a driving trend in terms of reduced traffic congestion and emissions in the future. Trucks from different logistics companies with a common interest can cooperate with the platoonbased pattern for cost-saving on goods transportation. For decision-makers and managers in the Ministry of
Transport, vehicle platooning can increase intersections' and ramps' capacity by decreasing headway. However, many factors affect the uptake of vehicle platooning in highways, such as vehicle types, vehicles from different companies, and higher fuel consumption of heading vehicles. A strategy to satisfy all vehicles' interests in a platoon is needed for transporting goods.

(4) Cloud-edge collaboration

Cloud-edge collaboration, namely effective collaboration between cloud computing and edge computing, is a promising computing scheme to cope with the massive data traffic [7]. In such a scheme, some delay-sensitive computation tasks of vehicles, such as real-time control information and road information, can be processed at the corresponding edge server close to the $5 \mathrm{G}$ base stations (gNB). Meanwhile, some delay-insensitive tasks (such as weather information and HD map) can be transmitted to the cloud server deployed at the cloud center for processing. By performing mobile computing, network control and storage at the network edge, mobile edge computing (MEC) is characterized by lower delay, energy-saving, privacy and security enhancement. Since cloud computing resources and communication capabilities are limited, a research trend on cloud-edge collaboration will consider how to obtain the computation balance. Thus, computation tasks can be processed appropriately by both the edge server and the cloud server. For this consideration, cloud computing and edge computing will cooperatively offload computation tasks and cache content to minimize the platoon-based MaaS system's overall latency. 


\section{(5) Service scheduling}

Service scheduling can provide vehicle-platoons with real-time, safe and efficient MaaS services for minimizing task completion time. Some tasks need to be migrated to edge servers or the cloud because vehicle-platoons have limited computing resources, which leads to some tasks that cannot be executed locally. These tasks require the same amount of time and computing resources for similar or different services provided by cloud-edge collaboration [8]. For example, latency constraints of different request messages (such as state monitoring information, control package and warning messages) on the Internet of Vehicles (IoV) can vary from milliseconds to seconds. Among these message categories, control packages require the lowest delay and have the highest priority to be executed to ensure the quality of service (QoS). Service scheduling is essential for the delay and QoS, especially when a flood of service requests come in. Thus, efficient scheduling and migration solutions are also a research focus for MaaS of vehicle platooning.

\section{Conclusion}

In general, a large and diverse collection of services in MaaS can maximize the benefits of the new digital road infrastructure, for which active research topics related to MaaS like $5 \mathrm{G}$, vehicle platooning, cloud-edge collaboration, and service scheduling will lead to more technological innovations.

Acknowledgements This work has been partially supported by the National Key Research and Development Program of China under Grant 2018YFE0197700.

\section{References}

1. Wong YZ, Hensher DA, Mulley C (2020) Mobility as a service (MaaS): charting a future context. Transp Res Part A Policy Pract 131:5-19

2. Smith G, Sochor J, Sarasini S (2018) Mobility as a service: comparing developments in Sweden and Finland. Res Transp Bus Manag 27:36-45

3. Allied Market Research report about truck platooning market. https://www.alliedmarketresearch.com/truck-platooning-market. Accessed 6 Nov 2020.

4. Jia D, Lu K, Wang J et al (2016) A survey on platoon-based vehicular cyber-physical systems. IEEE Commun Surv Tutor 18(1):263-284

5. Campolo C, Molinaro A, Iera A et al (2018) 5G network slicing for vehicle-to-everything services. IEEE Wirel Commun 24(6):38-45

6. Molina-Masegosa R, Gozalvez J (2017) LTE-V for sidelink 5G V2X vehicular communications: a new $5 \mathrm{G}$ technology for shortrange vehicle-to-everything communications. IEEE Veh Technol Mag 12:30-39

7. Ren J, Yu G, He Y et al (2019) Collaborative cloud and edge computing for latency minimization. IEEE Trans Veh Technol 68(5):5031-5044

8. Sharma V, Jayakody DNK, Qaraqe M (2020) Osmotic computingbased service migration and resource scheduling in Mobile Augmented Reality Networks (MARN). Future Gener Comput Syst 102:723-737

Publisher's Note Springer Nature remains neutral with regard to jurisdictional claims in published maps and institutional affiliations. 